\title{
Zweiter Teil, zweites Buch: Offenbarung oder die allzeiterneuerte Geburt der Seele
}

\author{
Gesine Palmer
}

\section{Abgrenzung \\ 1.1 Offenbarung philosophisch - Offenbarungsbegriffe in den realen Religionsgemeinschaften}

Rosenzweig hielt den zweiten Teil des Stern - und in ihm das zweite Buch für das Beste, was er geschrieben hat. Schon im Weiterschreiben am dritten Teil glaubte er, die Höhe des zweiten Buchs nicht wieder erreichen zu können. Tatsächlich laufen in diesem zentralen Stück verschiedene Fäden seiner Konstruktion zusammen. Es enthält - wenn man es durch die Brille heutigen Fachmenschentums lesen wollte - eine philosophische Theorie der primären Bindung und ihrer Bedeutung für das Wirklichkeitsgefühl des Menschen. Damit ist es aber zugleich auch etwas wie ein "dialogischer oder relationaler Gottesbeweis“ jenseits oder vor aller Ausdifferenzierung der verschiedenen Religionen. Zwar erläutert Rosenzweig seinen Begriff des wahren Glaubens und der Offenbarung auch in diesem Buch am Gegenbild des Islam und des Heidentums. Aber hier, sozusagen im Auge des Sternenorkans, geht es so konzentriert um die wechselseitige Hervorbringung von Gott und Mensch, dass die Grunddifferenz zwischen Judentum und Christentum fast völlig in den

How to cite this book chapter:

Palmer, G. 2021. Zweiter Teil, zweites Buch: Offenbarung oder die allzeiterneuerte Geburt der Seele. In: Brasser, M., Bojanić, P. and Ciglia, F. P. (eds.) The Star for Beginners: Introductions to the Magnum Opus of Franz Rosenzweig. Pp. 99-111. London: Ubiquity Press. DOI: https://doi.org/10.5334/bco.i. License: CC-BY 
Hintergrund tritt, während geradezu platt deutlich wird, wie sehr der Islam als eine Folie dient, um einen falschen Offenbarungsbegriff zu illustrieren. In der Realität der historischen Religionsgemeinschaften ist dieser in Rosenzweigs Augen falsche Offenbarungsbegriff selbstverständlich unter Christen und Juden genauso verbreitet wie im Islam - während umgekehrt auch im Islam dem kundigen Forscher Denker bekannt sind, die einen ähnlich elaborierten Offenbarungsbegriff haben wie Rosenzweig ihn für „den wahren Glauben“ beansprucht. ${ }^{178}$ Tatsächlich muss geradezu umgekehrt erst einmal festgestellt werden: Was Rosenzweig „Offenbarung“ nennt, würde vielen einfacher gläubigen Gemütern als das ganze Gegenteil von Offenbarungsglauben erscheinen. In einem resümierenden Satz sagt er es selbst so: „Die Offenbarung ist gegenwärtig, ja ist das Gegenwärtigsein selber" (SE 207; Hervorhebung GP).

\subsection{Theoretisierung und Metaphorisierung eines Liebesproblems}

Das Buch beginnt mit dem Satz „Stark wie der Tod ist die Liebe“ und springt mitten hinein in eine Situation zwischen Liebendem und Geliebter. Als zeitgenössische Leserin verschluckt man ein Entsetzen - oder vielleicht besser nicht. Es wäre letztlich ganz unrosenzweigisch, es zu verschlucken, und insofern auch für ihn keine angemessene Antwort. Das Buch versammelt schon auf der ersten Seite äusserst anstössige Sätze, von denen der folgende vielleicht am meisten empört: „Der Geliebten zunächst gilt es, dass die Liebe stark ist wie der Tod. Wie nur dem Weibe, nicht dem Manne die Natur es gesetzt hat, an der Liebe sterben zu dürfen“ (SE 174). Rosenzweig war in jenen Jahren von der Idee, dass eine junge Frau an der Liebe sterben und einen Opfertod auf sich nehmen könnte wie Alkestis, tief fasziniert. Das hatte einerseits sicher mit seiner eigenen Lage als Soldat zu tun, in der er gerade als Mann einem möglichen frühen Opfertod täglich gegenüberstand. Andererseits damit, dass er eine bedrängende und komplizierte biographische Situation durch eine weitere Komplikation "gelöst“ hatte: Er hatte sich in die Frau seines Freundes Eugen Rosenstock verliebt, der ihn immer wieder bedrängte, zum Christentum zu konvertieren wie er. Seit der ersten intimen Begegnung des Paares schrieb Rosenzweig Gritli eine grosse Zahl von Briefen. Die Liebe der beiden wurde offen ausgelebt, und viel besprochen, wobei auch immer wieder von der Liebe zwischen Eugen und Franz die Rede ist. Auf diese Geschichte ist das „Herzbuch“ vielleicht nicht zurückzuführen - aber sie sollte auch deswegen nicht unerwähnt bleiben, weil Rosenzweig selbst gerade dieses Buch in besondere Beziehung zu Gritli stellt. ${ }^{179}$

${ }^{178}$ Vgl. Einleitung (GP) und Nachwort (Yossef Schwartz), in Franz Rosenzweig: „Innerlich bleibt die Welt eine“. Ausgewählte Schriften zum Islam, Berlin/Wien 2003.

179 GB 177, Brief vom 2. Nov. 1918: „Dies Buch II 2, an dem ich jetzt schreibe gehört dir noch viel eigener als das Gritlianum, grade weil es nicht von 
In einem seiner etwas über 1000 erhaltenen Briefe an Gritli schreibt er von einer gemeinsamen Freundin Doris, diese sei an Gritlis Stelle „heruntergestiegen“ ${ }^{180}$ Die Vorstellung, dass eigentlich Gritli an der Liebe zwischen den beiden hätte sterben müssen, war ihm also nicht fremd.

Mit derselben Entschlossenheit, mit der ich diese Geschichte hier zu bedenken gebe, möchte ich freilich vor Kurzschlüssen warnen. Rosenzweig hielt sich selbst an seine ebenfalls in II.2 mitgegebene Kunsttheorie, in der die Einfälle, die aus dem Leben des Künstlers und über ihn kommen, sorgsam im Zuge der handwerklichen Arbeit von dem Leben und der Person abgelöst und zu einem Werk verarbeitet werden. Ich werde die Zusammenhänge noch näher beleuchten. Zu Beginn ist erst einmal wichtig, Probleme zu klären, die leicht verhindern könnten, dass man sich auf den tieferen Sinn des Textes einlässt.

Die Anmutung einer furchtbaren Pathetisierung der als natürlich ausgegebenen traditionellen Geschlechterordnung ist von diesen Hindernissen sicher eines der schwersten. Zugleich hängt gerade an diesem "Gleichnis“ die ganze Konstruktion, weit über die möglicherweise veranlassende reale Liebeserfahrung hinaus. Die Liebesbeziehung zwischen einem Liebenden und einer geliebten Seele in ihrer fundamentalen Asymmetrie erweist sich im Verlauf der in diesem Buch folgenden Ausarbeitung als eine hochdifferenzierte Darstellung dessen, was die „allzeiterneuerte Geburt der Seele“ ausmacht. Möge für die vorausgeschickten Abgrenzungen die Feststellung genügen, dass am Ende des Buches die geliebte Seele gerade nicht stirbt. ${ }^{181}$ Sie hat vielmehr erst Gott als den

vornherein für dich bestimmt war und es ja auch jetzt nicht ist. Es ist nicht ,Dir' aber - dein. Dein - wie ich. Manchmal ist mir, als wäre ich ein Kind, das nicht schreiben kann und es doch gern möchte und du führtest mir die Feder. Tu's weiter, Geliebte." Die Grundidee zu diesem Buch nannte er "Gritlianum“. An verschiedenen Stellen im umfassenden Briefwerk sagt er, dass er sie ursprünglich „der Schrei“ nennen wollte - ein Thema, das sich im hier verhandelten Buch auch wieder findet.

${ }^{180}$ GB 179, Brief vom 8. Nov. 1918: „Wir hatten freilich bei der Nachricht von Doris beide das gleiche Gefühl, deinen Brief bekam ich erst viel später. Aber ich meine, es war das Gefühl, als ob sie wie ein Opfer an deiner Stelle heruntergestiegen wäre. Ein furchtbares Gefühl, aber so kam es mir.“ Dazu zu lesen sind Erwägungen aus einem Brief vom 31. 10. 1918, in dem Rosenzweig über den Tod von Doris von Beckerath berichtet und wieder sehr allgemeine Überlegungen über das Verhältnis von Mann und Frau anstellt.

181 Es ist gelegentlich zu hören, dass in Wahrheit Rosenzweig an der letztlich unerfüllt gebliebenen Liebe zu Gritli gestorben sei. Dafür lassen sich jedenfalls dann Indizien finden, wenn man die Berichte über seine Verfassung nach seinem Entschluss zur Verheiratung mit Edith Hahn pathologisierend als den Beginn einer schweren Depression und den Ausbruch der ALS psychosomatisch liest. Ich warne aber auch hier vor Kurzschlüssen (für eine differenzierte Betrachtung vgl. mein Die Qual der Kreatur bewährt vermutlich nichts. Überlegungen zum Zusammenhang von Krankheit und 
Liebenden zu dem gemacht, was er ist, und „überschreitet“ den „Zauberkreis der Geliebtheit“, um nun ihrerseits den Mund zu öffnen, „nicht zur Antwort mehr, sondern zum eigenen Wort“ (SE 228). Insofern zeigt dieses Herzbuch im kleinen und für die weiblich imaginierte geliebte Seele dieselbe Struktur, die das ganze Werk für den Menschen überhaupt kennzeichnet: es beginnt mit der Todesfurcht und endet mit dem Entschluss oder der Bestimmung, ins Leben zu gehen.

\subsection{Gleichnis und Nichtgleichnis - das Hohelied als „Kernbuch der Offenbarung“"}

Die Idee des Buches ist extrem - das heisst: in voller Konsequenz und präziser sprachlicher Durchführung - prozesshaft. An keiner Stelle dürfte das deutlicher sein als da, wo Rosenzweig über den Gleichnischarakter desjenigen biblischen Buches spricht, das er als „Kernbuch der Offenbarung“ (SE 225) ansieht. Der Prozess, um den es hier geht, ist der einer gleichsam dynamischen mystischen Vereinigung, aus der die Realität des einen im Glauben an die Realität des anderen hervorgeht. Unio mystica - mystische Vereinigung - wird also nicht als Endstadium eines kontinuierlichen Loslassens irdischer Bindungen verstanden, in dem dann die endgültige Gelassenheit der reinen Seele erreicht wäre. Vielmehr bedeutet sie gerade umgekehrt die Entstehung der Vielfalt der weltlichen Bindungen aus der wechselseitigen Veränderung der Verschiedenen in der Liebesbeziehung. In der Geschichte der Bibelforschung hat man sich immer wieder mit der Frage beschäftigt, warum ausgerechnet das Hohelied, das doch alle Charaktere eines „weltlichen“ Liebesliedes trägt und nirgends von Gott spricht, in den Kanon der heiligen Schriften aufgenommen wurde. Rosenzweig beantwortet die Frage damit, dass er sagt: nur dieser Text behandelt die Liebe nicht mehr als ein Gleichnis, wie dies die Prophetenbücher tun, in denen immer wieder die eheliche oder aussereheliche „sündige“ Liebe als Gleichnis für die Beziehung zwischen Gott und seinem Volk verwendet wird. Dabei verlässt Rosenzweig die mystischen Deutungen, in denen man die Vereinigung eines präexistent gedachten Gottwesens und eines nachgeordnet präexistenten Menschenwesens sehen könnte, und lässt das Selbstsprechen als die Weise hervortreten, in der das Seelewerden sich allein ausdrücken und offenbaren kann. „Es gibt kein Buch in der Bibel, in dem verhältnismässig das Wort Ich so häufig vorkäme wie hier“ (SE 225) - gerade dadurch qualifiziert das Hohelied sich für Rosenzweig zum Kernbuch der Offenbarung. Mit der Überwindung der Sterblichkeit durch die Liebe im Eröffnungssatz „Stark wie der Tod ist die Liebe“ ist also nicht gemeint, dass irgendwelche entstofflichten Entitäten unsterblich wären, während der Leib stirbt, und es geht Rosenzweig auch nicht um die

Denken Franz Rosenzweigs, in: M. Brasser (Hg.), Rosenzweig Jahrbuch $1 /$ Rosenzweig Yearbook 1. Rosenzweig heute / Rosenzweig today, Freiburg/ München 2006, 232-254. 
Vorstellung einer leiblichen Auferstehung. Sein Anliegen in aller Rede von der Liebe, die mehr wäre als ein Gleichnis, geht auf den Augenblick aus, in dem die selbstoffenbarende Ichrede aussetzt. Das wird im folgenden noch ausgeführt werden. Hier ist erst einmal festzuhalten, dass die Voraussetzung dieses Augenblicks ebenso wie die Voraussetzung seiner Dauer immer die Befreiung der lebendigen Sprache aus aller die Menschenseele (oder: Gott, Welt und Mensch) verobjektivierenden Rede der Philosophie und der „falschen Offenbarung“ ist. Es geht um das „Sagen“ der Beziehung in allen ihren „Reifestadien“ (wie man sie nur in wissenschaftlich verdinglichter Form darstellen könnte) nicht um eine objektivierende Darstellung verschiedener möglicher Zustände von Menschen- und Gottesbeziehungen. Die sprachliche Höhe dieses Sagens sieht Rosenzweig im Hohelied erreicht. Dieses spricht die Gegenwart einer Beziehung reiner aus als alle Verkündigungen mit ihren Gleichnissen es vermöchten. Der Augenblick, in dem die Ichrede aussetzt, gilt Rosenzweig als derjenige Augenblick, in dem die Offenbarung sich mit der Schöpfung verbindet (SE 225). ${ }^{182}$

\section{Momenthafte Selbstverwandlung, nicht Eigenschaft}

\subsection{Die Liebe des Liebenden und die Liebe der Geliebten}

In den ersten Absätzen von II.2 knüpft Rosenzweig an II.1 an, um zu erläutern, inwiefern innerhalb seines Systems ${ }^{183}$ die Schöpfung notwendig mit einer Offenbarung verknüpft sein muss. Dabei geht es nicht nur um die Frage nach der Wirklichkeit Gottes - oder die Frage, wie rational es sein kann, einen wirklichen Gott denken zu wollen. Es geht vielmehr um die Frage nach Wirklichkeit

182 „Das Wort ,Ich' also ist der Grundton, der bald in der einen, bald, durch das Du übergehend, in der anderen Stimme unter dem ganzen melodisch-harmonischen Gewebe der Mittel- und Oberstimmen orgelpunktartig hinzieht. Es gibt in dem ganzen Buch nur eine einzige Stelle, wo er schweigt; sie fällt gerade durch dieses augenblickliche Aussetzen des Grundbasses, den man sonst in infolge seiner Unaufhörlichkeit schon beinahe überhört, ungeheuer auf; so wie man sich des Tickens der Wanduhr erst bewusst wird, wenn sie plötzlich stillsteht. Es sind die Worte von der Liebe, die stark ist wie der Tod.[...] In ihnen ragt die Schöpfung sichtbar in die Offenbarung hinein und wird sichtbar von ihr überhöht. Der Tod ist das Letzte und Voll-endende der Schöpfung - und die Liebe ist stark wie er.“

${ }^{183}$ Dass es sich schliesslich um nichts Geringeres als ein „System der Philosophie" handele, hat er bereits während des Schreibens in seinen Briefen und später dann auch in den auf den Stern bezogenen Essays deutlich ausgesprochen, und viele Forscher haben mit graphischen Darstellungen des Sternsymbols den Systemcharakter erläutert - dies alles hier zu wiederholen wäre also überflüssig. 
überhaupt - und wie diese sich verändert, wenn wir in unserem Denken von Aktivität und Passivität mit der Ereignishaftigkeit von Sprache und menschlicher Begegnung ernstmachen. Übersetzt in die Sprache des gegenwärtig vorherrschenden (und darum als allgemeinverständlich geltenden) Psychologismus würde man sagen müssen: Es geht um die Frage nach der („gefühlten“) Wirklichkeit emotionaler Verbindungen überhaupt. ${ }^{184}$

Der Tod - dies ist in den vorigen Kapiteln des Werkes überdeutlich geworden - gehört zur Schöpfung. Er ist, wie Rosenzweig in diesem Kapitel sagt, „das Letzte und Volle-endende der Schöpfung”. Nun wird also gesagt, „die Liebe ist stark wie er”, und Rosenzweig fährt fort: „Dies ist das einzige, was über die Liebe gesagt, aus-gesagt, erzählt werden kann; alles andere kann nicht, über' sie gesagt ,werden', sondern nur von ihr selber gesprochen“" (SE 225).

Wie man sich aber das Sprechen der Liebe gegenüber dem Tod als ein Sprechen in menschlicher Sprache vorstellen könne, davon handeln die Schematismen, mit denen Rosenzweig die Liebe zwischen der Liebe des Liebenden und der Liebe der Geliebten aufteilt. Wenn wir seiner Metaphorik zunächst nicht in das Gleichnis der Liebe zwischen Mann und Frau folgen, sondern - in Parallele zur späteren Entwicklung in der Psychoanalyse - erst einmal auf die Beziehung zwischen dem abhängigen, seine Bedürftigkeit herausschreienden Menschensäugling und seiner ersten (zumeist mütterlichen) „Bezugsperson“ zurückgehen, können wir vieles sehr viel deutlicher verstehen. So wie die spätere Psychoanalyse die Bedürftigkeit auch der Mutter anerkennt, so formuliert

${ }^{184}$ Es ist vermutlich kein Zufall, dass die Arbeiten Freuds und die Arbeiten Rosenzweigs etwa zeitgleich entstanden. Das Problem der Offenheit menschlichen Fühlens und der dieses ,einhegenden“ kulturellen Definitionen war in der Zeit um den Ersten Weltkrieg durch die industrielle Entwicklung und die damit verbundene Erosion der alten Ordnungen bis zu ihrem völligen Scheitern in diesem Krieg so eklatant ins Bewusstsein der gebildeten Zeitgenossen gedrungen, dass alles für sicher gehaltene Sprechen über die "natürlichen menschlichen Beziehungen“ nicht mehr als gültig empfunden werden konnte. Aus der Sicht , aufgeklärter Zeitgenossen“ nahm Rosenzweig den „Rückweg“ in die Religion, wenn auch in eine sehr aufgeklärte Variante davon, während Freud vorwärts ging in die Wissenschaft. Von heute aus stellt sich das insofern etwas anders dar, als die verdinglichende Rede vom Menschen umso deutlicher an ihre Grenzen stösst, je weiter auch die physiologische Erforschung der Vorgänge im menschlichen Gehirn voranschreitet. Werke wie Der Stern der Erlösung reden da nicht dem Rückfall in mythisch-religiöse Erklärungen das Wort, sondern erlauben ein modernster Entwicklungs- und Sprachtheorie angemessenes Systematisieren derjenigen Sprachen, in denen wir auf das Paradox, gleichzeitig Forscher und Gegenstände unserer Forschungen zu sein, antworten und aus der isolierenden Stillstellung, die eine pseudoobjektive, vermeintlich rein wissenschaftliche Rede uns antun würde, ausbrechen. 
dieses theologische oder philosophische System etwas wie ein Bedürfnis Gottes nach dem Menschen. Um dies gegen die Tradition zu rechtfertigen, verweist Rosenzweig wieder auf die Prozesshaftigkeit des dialogischen Offenbarungsgeschehens. Auf Rosenzweigs Dynamisierung der traditionellen Begriffe komme ich gleich zurück.

Zunächst einmal will ich begründen, warum ich glaube, dass die Beziehung zwischen Mutter und kleinem Kind besser geeignet wäre als die erotische Beziehung zwischen Erwachsenen, um die Figur der den anderen hervorbringenden und das Selbst offenbarenden Liebe zu symbolisieren. Die Kampfansage der Liebe an den Tod, das Schmachten der Liebe nach Gegenwart, sind selbstverständlich in allen Liebesformen anzutreffen. Aber dieses spezielle Wandelnlernen aus der Gewissheit des Geliebtseins, von dem Rosenzweig schreibt, dieses Beimnamengerufensein, dieses erst Festwerden unter der Antwort auf das Liebmich des Liebenden, erinnert doch mehr an das Ichwerden des kleinen Kindes, das von der Mutter beim Namen gerufen wird und sich in dem Masse, in dem es selbst antworten lernt, dann auch von der Mutter entfernen und selbstsprechend in die Welt gehen kann, ohne den Kontakt zur Mutter zu verlieren. Dass es dabei wegen seiner unendlich grösseren Abhängigkeit und Angewiesenheit auf die liebevolle Zuwendung der Mutter auch das Wesen ist, das aus Liebe sterben könnte, liesse sich auf sehr traurige Weise an den vielen Fällen illustrieren, in denen Kinder eher ihr Leben als die Beziehung zu peinigenden Eltern aufgeben. Während umgekehrt das Gelingen einer jeden Liebesbeziehung zwischen Eltern und Kindern nach dem Wunder der Entstehung des Kindes in Zeugung und Geburt die eigentliche, allzeiterneuerte Offenbarung der Menschlichkeit ist. Dazu gehört auch der Charakter der Bedürftigkeit und des Überwältigtseins auf Seiten des Liebenden. ${ }^{185}$

${ }^{185}$ Diese Frage wird immer noch besonders differenziert diskutiert in dem Kapitel „Das verlorene Ideal der Mütterlichkeit“ in Jessica Benjamins Die Fesseln der Liebe. Psychoanalyse, Feminismus und das Problem der Macht, Frankfurt a.M. 1993, woraus hier nur ein kleiner Absatz zitiert sei: „Das Kind kann die Mutter nur dann als Subjekt mit eigenem Recht wahrnehmen, wenn die Mutter auch ein solches ist. Und hier sollten wir uns klarmachen, dass zur Subjektivität der Mutter (kontrastiert zum Ideal der Mütterlichkeit) auch Unvollkommenheit gehören muss, damit sie für sich selbst und für ihr Kind real sei” (207), es lohnt sich aber, den ganzen Text einmal neben Rosenzweigs zu halten. Obwohl Benjamin philosophisch eher auf einen Diskurs der gegenseitigen Anerkennung rekurriert, liefert sie doch genau das Mass an psychoanalytischem Basiswissen über die Elemente der asymmetrischen Beziehung zwischen Erwachsener und Säugling bzw. Kleinkind, die gewusst werden müssen, wenn man die Entwicklung vom unbedürftigen allerbarmenden Gott zum bedürftigen liebenden Gott auf einer passenden irdisch-zwischenmenschlichen Grundlage verstehen will. 


\subsection{Das Nichts und die innere Umkehr}

Wenn die Prozesshaftigkeit der Liebesoffenbarung - an welchem lebensnahen Beispiel einer asymmetrischen Beziehung auch immer - anschaulicher geworden ist, kann nun noch einmal die abstraktere Gestalt des „dynamischen Gottesbeweises" in den Blick genommen werden. Die Konstruktion des Stern hängt einerseits an der graphischen Figur das Davidsterns und damit auch an der Idee, die Beziehung von Gott, Mensch und Welt, von Schöpfung, Offenbarung und Erlösung more geometrico darzustellen, wie dies für philosophische Systematiker nicht erst seit Spinoza und nicht nur bis Hegel verlockend war. Andererseits soll der Stern, wie Rosenzweig in einem seiner Briefe über seine „Vision“ von der Sache berichtet, rollen. Und drittens - soll das Ganze auf lebendigere Weise als frühere Entwürfe „offen“ sein oder ins Offene, ins Lebendige hinausführen, und zwar, so Rosenzweigs Anspruch, ausdrücklich ohne vorprogrammiert, zugleich aber auch ohne beliebig zu sein. Genau diesem Anspruch nähern sich Gott, Welt und Mensch in der Offenbarung. Rosenzweig kennzeichnet sie als eine „Zweite“ Offenbarung. In der ersten, der Schöpfung, war Gott von einer Selbstverneinung des Nichts zu einer Weltbejahung gelangt. Diese Weltbejahung, so sagt Rosenzweig am Anfang des zweiten Buches, war aber nur eine abermalige Verneinung seiner eigenen Lebendigkeit, ein Nichts höherer Stufe, „Nichts nur mit Bezug auf das, was ihm entsprang, in sich aber ein Nichts voller Charakter, eben kein Nichts, sondern ein Etwas. Nichts war sie nur darin, dass sie, indem sie sich auftat, sofort in neue Gestalten auseinanderbrach" (SE 175). In diesem Zusammenhang nun redet Rosenzweig selbst von Gott als einem mütterlichen Wesen, dessen ,innere ,Natur', die unendliche Meeresstille seines Seins", auf Offenbarung drängt - oder ihrer bedarf.

Es fällt mir schwer, hier nicht entweder einfach nachzuerzählen - oder sehr kritisch zu kommentieren. Spekulationen über Seinsvorgänge in mystischen Urgründen haben der Rationalistin immer etwas Raunendes, Spökenkiekerisches, wenn sie nicht einfach nur eine sehr metaphorische Rede sein sollen. Hinter die Feststellung, dass Rosenzweig selbst die Notwendigkeit der inneren Umkehrung gesetzt hat, komme ich nicht zurück, auch dann nicht, wenn ich dreissig mal die Stellen lese, an denen er in der ersten Lebendigkeit Gottes den geometrischen Punkt bezeichnet sieht, aus dem nun der Hervortritt Gottes in die Offenbarung erfolgen müsse. Dies aber einmal gegeben, erkennt man an den Gegenüberstellungen zu Islam und Heidentum schnell, worauf dieses Umkehrungstheorem hinausläuft: Es versucht mit allen Mitteln der Theologie und der Philosophie, Gott und Mensch in eine lebendige Beziehung zu ziehen und dieses Lebendige selbst mehr zu sagen als nach dem Muster einer Begriffsreihe oder Kausalverknüpfung zu erklären. Dennoch soll die Rationalität in Geltung bleiben. Der „wahre Glaube“ soll nicht nach Art der geheimnisvollen oder mythischen Religion funktionieren und einfach eine der Erklärung nicht weiter bedürftige Behauptung aufstellen, wie es die buntbebilderte Götterwelt der Heiden tue. Das Verborgene der Macht hinter der vorgefundenen Schöpfung soll dem wahren Glauben gerade stark bewusst und fühlbar sein. Die Feststellung, dass die Schöpfung gut war, „gar sehr“, war am Ende von II.1 
zugleich der Tod, denn es war eine Feststellung über ein ein für allemal Gegebenes. Rosenzweig nennt es dunkel oder verborgen. Licht nennt er die augenblickshaft hereinbrechende oder aus Gott hervorbrechende Offenbarung, und sie ist in seiner Metaphernwelt zuerst etwas Väterliches: „Das Mütterliche ist stets das, was schon da ist, das Väterliche kommt erst hinzu“" (SE 177). Und als hätte er sämtliche spätere Lehrbücher der Psychoanalyse schon mal vorab gelesen, kommt dieses Spätere hinzu als Namengebung und Anrede. Wieder ist es ganz unmöglich, die Briefe zu übergehen, die Rosenzweig in diesem Zusammenhang schrieb. Etwa diesen Briefanfang vom 15.08.1918:

„Liebes Gritli,

-ich sitze ganz stumm vor den zwei Worten und möchte weiter gar nichts schreiben, habe auch eigentlich gar nichts weiter zu schreiben, und habe ich dir denn eigentlich je etwas andres geschrieben?" (GB 118)

Ich werde nicht von Bewährung reden. Ich werde nicht die Briefe auf den Stern oder den Stern auf die Briefe zurückführen. Ich werde auch die Zeitproblematik nicht schärfer stellen als Rosenzweig selbst es im Stern tut (obwohl ich den Eindruck habe, man müsste das). Festgehalten sein soll nur: Die Erschaffung der Welt ist für Rosenzweig etwas, was Gott aus sich heraussetzt, was aber auch „in ihm selber als dem verborgenen vorgeht“. Symptomatisch dafür ist ihm das göttliche Selbstgespräch des „lasst uns einen Menschen machen”. Nun könnte er in die Dunkelheit seiner Verborgenheit oder des Nichts, aus dem heraus er die Welt erschaffen hat, zurücksinken. Dann wäre die Schöpfung da: als ein „Ding“ und als insofern stummes Zeugnis der ersten, der noch fast uneigentlichen Offenbarung. Und Gott wäre wieder verborgen. (Übertragen auf die Situation zwischen einer Mutter und einem Kind könnten wir sagen: das Kind wäre geboren, könnte aber die Nähe zur Mutter jederzeit wieder verlieren, wie die Mutter mit dem Kind ihr Muttersein verlieren könnte). Eine Offenbarung, die ihn am hellen Licht hielte, wäre aber diejenige, die nicht durch Selbstverneinung des göttlichen „Wesens“ ein „Ding“ setzte. Sie müsste vielmehr eine Beziehung zu dem nun stumm vorhandenen Ding beginnen und also nicht wieder etwas Neues erzeugen, sondern „nichts sein als das Sichauftun eines Verschlossenen, nichts als die Selbstverneinung eines blossen stummen Wesens durch ein lautes Wort, einer still ruhenden Immerwährendheit durch einen bewegten Augenblick“ (SE 179).

Mit diesen systemischen Anforderungen an das, was eine Offenbarung innerhalb des geometrischen Schemas und in ihrer Mitte zu leisten hätte, leitet Rosenzweig zu seinem persönlichen, philosophischen Hohelied der Liebe über.

\subsection{Die Theodizee-Arbeit der geliebten Seele}

Aus der in Gott selbst begründeten Notwendigkeit einer inneren Umkehr folgt nun eine weitere Umkehrung, die ich mit Bedacht nicht mehr unter der Überschrift der „inneren“ Umkehr behandeln möchte. Es kommt nämlich in dem in 
eigentümlichen Tempi beschriebenen Verhältnis von Gott als dem Liebenden und der menschlichen Seele als der Geliebten zu eigenartigen Umkehrungen nicht nur der traditionellen Begriffe in sich, sondern der Verhältnisse selbst, innerhalb der Begriffe, innerhalb der Seelen der Beteiligten und zwischen ihnen. (Das wird in den folgenden Büchern dann auch Folgen haben für die Umkehrungen in den Verhältnissen der Religionen zueinander).

Es beginnt mit der Liebe des Liebenden. Diese muss augenblickshaft sein und bleiben. Sie ist treulos in ihrem Wesen, weil ihr Wesen der Augenblick ist, der allein sie zum Anderen für den bisherigen göttlichen Stillstand machen kann. Sie ist seine Verbindung zu Zeitlichkeit und Lebendigkeit. Der Liebesaugenblick kann aber (das ist die innere Umkehrung in der Idee der Liebe des Liebenden) aus dem Element der tiefsten Untreue zur Voraussetzung für die beständige Treue werden, mit der der Liebende die Geliebte täglich ein bisschen lieber hat. Die so verstandene Liebe steigert sich, weil sie immer neu sein will. Dennoch ist sie nicht nur eine flüchtige Wallung. Ich gehe nicht weiter in die Nacherzählung der Elemente. Es geht hier vor allem um die Konstruktion der Verbindung von stiller Ewigkeit und lebendiger Zeitlichkeit. Interessant daran ist, dass diese so sehr augenblickshafte, von Augenblick zu Augenblick sich wieder offenbarende Liebe des Liebenden eben die geliebte Seele braucht. Wenn auch nur, um etwas zum Lieben zu haben. Sie ist unberechenbar und punktuell, durchwandert aber das ganze All. Am Gegensatz zum Islam - wie er ihn sieht - erläutert Rosenzweig, wieso sie nun nach und nach ergreifen muss, aber jedes einzelne in dem jeweiligen Augenblick so, dass sie darüber alles andere vergisst. Der - systematische - Grund besteht in der besonderen Verbindung, die durch die Antwort der geliebten Seele erst zu einer Verbindung wird. Die Seele wird von dem Augenblick, in dem die Liebe des Liebenden sie ergreift, „überschauert”, wie Rosenzweig schreibt. Was bisher Trotz war, wird nun dieselbe Eigenschaft, die sie befähigt, diesen Augenblick dauernd und gleichmässig festzuhalten. So dass sie - die sterbliche - vom Modus des zu sehr Bewegten in den Modus der Ruhe übergeht. Es ist, wenn man so will, ein Austausch der Elemente von Festigkeit und Bewegtheit. Wobei die geliebte Seele zunächst diejenige bleibt, die sich daran genügen lässt, das Geliebtwerden zu empfangen, sich für es zu öffnen und es festzuhalten als den Liebesglanz des eigenen Lebens, dessen Vergangenheit vor diesem Augenblick zu nichts wird.

Natürlich liegt in seinen wortreichen Schilderungen dieser Aspekte der Liebe zwischen dem liebenden Gott und der geliebten Seele die Metaphorik der erotischen Liebe zwischen Mann und Frau am nächsten. So sehr, dass Rosenzweig selbst sich genötigt sieht, einer zu direkten Übertragung eine Grenze zu setzen, indem er sagt, dass „die Rollen des Liebe Gebenden und Liebe Empfangenden hin und her [gehen], obwohl von den Wurzeln der Geschlechtlichkeit her sich immer wieder das eindeutige Verhältnis der Natur wiederherstellt" (SE 189). Dennoch glaube ich, man kann es fast anschaulicher machen, wenn man wieder die Situation zwischen der Mutter und dem sehr kleinen Kind ins Auge fasst. Dieses ist wirklich in sehr hohem Masse für sein Leben darauf angewiesen, 
dass die Eltern sich ihm immer wieder neu zuwenden, es beim Namen nennen, ihm überhaupt einen Namen geben, durch den sie es aus dem Diffusen ebenso herausrufen wie aus dem bloss Gegenständlichen. Auf das kleine Kind trifft zu, dass es wirklich nicht war, bevor es in die elterliche Liebe geholt wurde. Und auf das erste Ichsagen des kleinen Kindes trifft in besonderem Masse zu, was Rosenzweig über das Ich überhaupt sagt: dass es „stets ein laut gewordenes Nein“, ein „Ich aber“ ist.

Rosenzweig geht nun den Urdialog zwischen Gott als Liebendem und dem Menschen als geliebter Seele durch. Der Gott der Gebote sagt „Ich der Ewige“ und redet als solcher den Menschen mit „Du“ an. Das Grundgebot Gottes lautet aber: „Liebe mich.“ Und die geliebte Seele antwortet darauf: „Ich habe gesündigt." Von Rosenzweig übersetzt als das beschämte Eingeständnis, dass sie vor dem Hören dieses Anrufes durch den Liebenden ohne Liebe war. Sie bekennt aber nicht nur die Liebeleere der Vergangenheit, sondern vor allem, dass sie „auch in diesem gegenwärtigsten der Augenblicke noch lange nicht so liebt, wie sie sich geliebt weiss". Wieder erscheint mir die Analogie mit dem Selbstgefühl des von einem Schutz gebenden und bergenden Erwachsenen geliebten Kindes evident.

Mit diesem elementaren Dialog aber verändern sich die Verhältnisse. Das Bekenntnis der geliebten Seele zu dem, von dem sie sich geliebt weiss, ist schon ein Bekenntnis Gottes. Das Vertrauen der geliebten Seele gegenüber dem liebenden Gott macht diesen erst offenbar. Rosenzweig geht weiter: Durch das Bekenntnis holt die Seele Gott nicht nur ins „Diesseits seiner Verborgenheit“, sondern: „Indem die Seele vor Gottes Antlitz bekennt und damit Gottes Sein bekennt und bezeugt, gewinnt auch Gott, der offenbare Gott, erst Sein: ,wenn ihr mich bekennt, so bin ich." Dafür wiederum gibt Gott der Seele eine Vergangenheit: „Ich habe dich bei deinem Namen gerufen. Du bist mein.“ Damit gibt das grundlos als Beziehung in die Welt der Dinge hineingerufene Gotteswort der Seele ihrerseits einen Grund in der Beziehung. Wieder ist die Folge für „den Glauben“ dieselbe wie in der gelingenden primären Bindung zwischen Mutter und Kind: „Gewiss, schon vorher konnte ihn nichts von Gott scheiden, aber doch nur, weil er in seiner Vertiefung in Gegenwärtiges nichts ausser sich sah. Jetzt darf er ruhig die Augen öffnen und um sich schauen in die Welt der Dinge...Die Seele kann mit offenen Augen und ohne zu träumen sich in der Welt umtun; immer bleibt sie nun in Gottes Nähe. Das ,du bist mein', das ihr gesagt ist, zieht einen schützenden Kreis um ihre Schritte. Sie weiss nun, dass sie nur die Rechte auszustrecken braucht, um zu fühlen, dass Gottes Rechte ihr entgegenkommt. Sie kann nun sprechen: mein Gott, mein Gott. Sie kann nun beten.“ (SE 205). Für den Augenblick, den eigentlichen „Punkt“ des Offenbarungsgeschehens ist das schon alles. Das Gebet selbst ist - wieder anders als in der heidnischen Vorstellung - die Erfüllung. „Sie betet um das Betenkönnen, das mit der Gewissheit der göttlichen Liebe ihr schon gegeben ist." So ist das in der Gegenwart des Offenbarungsaugenblick. Für die Beziehung der geliebten Seele zu Gott scheint nun alles geklärt. Aber noch geht es nur um 
Betenkönnen und Betenmüssen - so wie es zuvor bei Gott um Liebenkönnen und Liebenmüssen gegangen war, das sich aber noch irgendwohin ergiessen musste, so geht es der Seele nun auch. Aus der passiven Aktivität Gottes und der aktiven Passivität der Seele kommt etwas Neues hervor, denn der Augenblick der Wechselrede mit Gott bleibt nicht stehen. Es gibt noch die Beziehung der menschlichen Seele zur Welt. In dieser gibt es Zukunft, und für diese hat die Seele einen Wunsch, eine Frage, einen Schrei. Hier scheint jetzt meine Veranschaulichung mit der seelischen Entwicklung des Kleinkindes in der Beziehung zur Elternperson nicht mehr aufzugehen.

Rosenzweig drückt sich da jedenfalls recht eindeutig erotisch aus: „Denn der Schrei, den die Seele im Augenblick der höchsten unmittelbaren Erfüllung stöhnt, tritt über die Schranken der Wechselrede hinaus; er kommt nicht mehr aus der seligen Gestilltheit des Geliebtseins, sondern steigt in neuer Unruhe aus einer uns noch unerkannten Tiefe der Seele und schluchzt über die ungesehene, doch gefühlte Nähe des Liebenden hinweg in den Dämmer der Unendlichkeit hinaus." (SE 206) Mit einer abermaligen konzentrierten Zusammenfassung des Sprachgeschehens, der Namengebung, des Gebots und der sich sagenden Annahme und mit einem Umweg über eine in sich ponderable Theorie des Genies, nähert sich Rosenzweig wieder dem Hohelied. Er paraphrasiert: „Die Geliebte fleht, der Liebende möge den Himmel seiner allzeitlichen Gegenwärtigkeit zerreissen, der ihrem Sehnen nach ewiger Liebe trotzt, und $\mathrm{zu}$ ihr herniederfahren, auf dass sie sich ihm wie ein ewiges aufs immerzuckende Herz legen kann und wie ein fest umschliessender Ring auf den nimmerrastenden Arm“ (SE 228). So übersetzt er den Satz „O dass du mein Bruder wärest”. Und in den letzten Absätzen scheint es sich zunächst wirklich fast 1:1 um eine Anrede an Gritli zu handeln: Das Reich der Brüderlichkeit, nach dem die Seele sich sehnt, „stiftet ihr nicht mehr die Liebe des Liebenden, von der sie bisher stets das Stichwort erwartet hatte, um Antwort zu geben. Soll dieser Sehnsucht Erfüllung werden, so muss die geliebte Seele den Zauberkreis der Geliebtheit überschreiten, des Liebenden vergessen und selber den Mund öffnen, nicht zur Antwort mehr, sondern zum eigenen Wort. Denn in der Welt gilt nicht das Geliebtsein, und das Geliebte darf es hier nicht anders wissen, als wäre es allein auf sich angewiesen und ungeliebt, und alle seine Liebe wäre nicht Geliebtheit, sondern ewig - Lieben. Und nur im geheimsten Herzen mag sie bei diesem ihrem Gang aus dem Wunder der göttlichen Liebe heraus in die irdische Welt der Alten Wort bewahren, das dem, was ihr zu tun bevorsteht, durch die Erinnerung des in jenem Zauberkreis Erlebten Kraft und Weihe gibt: Wie er dich liebt, so liebe Du." (SE 228)

Damit endet das zweite Buch. Im Kern machen Seele und Gott einander wirklich, indem sie diesen sehr dynamischen Urdialog, den Gott durch sein Beim-Namen-Nennen initiiert, miteinander führen, bei dem jeder sich verändert. Ohne diese Offenbarungsbeziehung zwischen Mensch und Gott wäre Gott ein Gott mit Eigenschaften, zu denen ein ihn selbst überwältigendes Verlangen nach Liebe zu seinen und von seinen Geschöpfen niemals gehören 
dürfte. Und der Mensch wäre ein Ding, das man unter Begriffe bringen könnte, die ihm aber kein Haus zum Wohnen sein würden, sondern, so schreibt Rosenzweig, sein zuständiges Gerichtsgebäude, zu dem er nur gerufen würde, um vernommen zu werden - was bei ihm zu den üblichen Ausreden, das Weib, die Schlange, der Apfel, führen würde. Durch die Liebe und das Geliebtsein werden beide lebendig und wirklich. Das als Mängelwesen nach Liebe dürstende Menschenwesen ist gerade in seiner Schwäche liebenswert, und auch Gott ist dem Menschen als Liebender gewiss, weil er selbst sich als liebender offenbaren muss, wenn er einmal bekannt wurde.

Vermutlich kann man so etwas nur schreiben, wenn man es erstens überhaupt kann, und wenn man zweitens - wie Rosenzweig es zur Zeit der Herstellung dieses Textes war - emotional so tief berührt ist, dass alle Akkorde aus der primären Bindung wieder anklingen, wo man selbst liebt. Nur dann ist das Buch selbst wie das Hohelied, von dem es handelt, Gleichnis und zugleich mehr als ein Gleichnis: ein Sichsprechen.

Damit, wie die so zuinnerst erfahrene Liebe nun in die Welt geht, beschäftigt sich das dritte Buch, und es geht allmählich vom unmittelbaren Glühen der sich sprechenden Begegnung wieder in ein Erklärendes über. Mein Beitrag aber endet hier.

\section{Weiterführende Lektüre}

Fricke, M., (2003), Franz Rosenzweigs Philosophie der Offenbarung. Eine Interpretation des Sterns der Erlösung, Würzburg, Königshausen und Neumann. Joskowicz, A.A., (2008), Rosenzweigs Konzept der Offenbarung und die Zeichen der Liebe, in: Naharaim 12 (2008) 164-187.

Rühle, I., (2004), Gott spricht die Sprache der Menschen: Franz Rosenzweig als jüdischer Theologie - eine Einführung, Tübingen, Bilam Verlag. 